\title{
Exact solution of inverse-square-root potential $V(r)=-\frac{\alpha}{\sqrt{r}}$
}

\author{
Wen-Du Li ${ }^{a}$ and Wu-Sheng Dai ${ }^{a, b, 2}$ \\ ${ }^{a}$ Department of Physics, Tianjin University, Tianjin 300072, P.R. China \\ ${ }^{b}$ LiuHui Center for Applied Mathematics, Nankai University $\& 3$ Tianjin University, Tianjin 300072, \\ P.R. China
}

ABSTRACT: An exact solution of the three-dimensional spherical symmetry inverse-squareroot potential $V(r)=-\frac{\alpha}{\sqrt{r}}$, including scattering and bound-state solutions, is presented.

\footnotetext{
${ }^{1}$ daiwusheng@tju.edu.cn.
} 


\section{Contents}

1 Introduction 1

2 Radial equation $\quad 2$

$\begin{array}{llr}3 & \text { Regular solution } & 2\end{array}$

4 Irregular solution $\quad 3$

4.1 Scattering boundary condition 3

4.2 Irregular solution 5

$\begin{array}{lll}5 & \text { Bound state and scattering state } & 6\end{array}$

5.1 Bound state 6

$\begin{array}{lll}5.2 & \text { Scattering state } & 7\end{array}$

6 Conclusion $\quad 8$

\section{Introduction}

The inverse-square-root potential $V(r)=-\alpha / \sqrt{r}$ is a long-range potential which has both scattering states and bound states. As a long-range potential, the inverse-square-root potential has a much longer range than the Coulomb potential. Its scattering state has a very different asymptotic behavior from that of the Coulomb potential. Its bound state, other than that of the Coulomb potential, has no closed classical orbits. An exact solution of the inverse-square-root potential enables us to investigate the behavior of long-range potentials deeply.

The inverse-power potential $V(r) \sim 1 / r^{s}$ with $0<s<2$ is a long-range potential and has both scattering states and bound states; while $V(r) \sim 1 / r^{s}$ with $s \geq 2$ is a shortrange potential and has only scattering states. The short-range power potential, $1 / r^{s}$ with $s \geq 2$, has only scattering states and can be generally treated [1, 2]. The long-range power potential, $1 / r^{s}$ with $0<s<2$, only when $s=1$, the Coulomb potential, is exactly solved. In this paper, we present an exact solution of the inverse-square-root potential — one other long-range potential.

The first thing we need to do is to determine the boundary condition, the value of the wave function on the boundary. At infinity, for scattering states, the wave function must equal the large-distance asymptotics; for bound states, the wave function must equal zero.

Different long-range potentials have different scattering boundary conditions, because the boundary condition of long-range-potential scattering at infinity is the large-distance asymptotic solution of the potential and, in general, the asymptotic solutions of different long-range potentials are different. Therefore, we have to determine the scattering boundary 
condition one by one for various long-range potentials, rather than short-range-potential scattering in which all potentials have the same scattering boundary condition. For this reason, in order to solve the inverse-square-root potential, a long-range potential, we need to solve its large-distance asymptotic solution first.

In section 2, we convert the radial equation of the inverse-square-root potential to the biconfluent Heun equation. In section 3, we solve the regular solution and in section 4 we solve the irregular solution. The exact solutions of bound and scattering states are given in section 5. The conclusion is given in section 6 .

\section{Radial equation}

The radial wave function $R_{l}(r)=u_{l}(r) / r$ of the inverse-square-root potential

$$
V(r)=-\frac{\alpha}{\sqrt{r}}
$$

is jointly determined by the radial equation

$$
\frac{d^{2} u_{l}(r)}{d r^{2}}+\left[k^{2}-\frac{l(l+1)}{r^{2}}+\frac{\alpha}{\sqrt{r}}\right] u_{l}(r)=0
$$

and the boundary conditions at $r=0$ and at $r \rightarrow \infty$.

At $r=0$, both for scattering states and bound states, the boundary condition is $u_{l}(0)=$ 0 (in fact, for the regular solution we use a stronger condition $\lim _{r \rightarrow 0} u_{l}(r) / r^{l+1}=1$ ). At $r \rightarrow \infty$, for scattering states, the boundary condition is $u_{l}(r \rightarrow \infty)=u_{l}^{\infty}(r)$, where $u_{l}^{\infty}(r)$ is the large-distance asymptotic solution of the radial equation (2.2); for bound states, the boundary condition is $u_{l}(r \rightarrow \infty)=0$.

By introducing $z=\sqrt{-2 i k r}$ and

$$
u_{l}(z)=A_{l} \exp \left(-\left(\frac{z^{2}}{2}+\lambda z\right)\right) z^{2(l+1)} f_{l}(z)
$$

where $\lambda=\alpha / \sqrt{2 i k^{3}}$ and $A_{l}$ a constant, we convert the radial equation (2.2) into an equation of $f_{l}(z)$ :

$$
z f_{l}^{\prime \prime}(z)-\left[2 z^{2}+2 \lambda z-(4 l+3)\right] f_{l}^{\prime}(z)+\left\{\left[\lambda^{2}-(4 l+4)\right] z-(4 l+3) \lambda\right\} f_{l}(z)=0 .
$$

The equation of $f_{l}(z)$, eq. (2.4), is just the so-called biconfluent Heun equation [3].

\section{Regular solution}

The regular solution is a solution satisfying the boundary condition at $r=0$. At $r=0$, the boundary condition for both bound states and scattering states is [4]

$$
\lim _{r \rightarrow 0} \frac{u_{l}(r)}{r^{l+1}}=1
$$

because the asymptotic solution of the radial equation (2.2) at $r=0$ is $u_{l}(r) \stackrel{r \rightarrow 0}{\sim} r^{l+1}[5]$. 
The biconfluent Heun equation, eq. (2.4), has two linearly independent solutions [3],

$$
\begin{aligned}
& y_{l}^{(1)}(z)=N\left(4 l+2,2 \lambda, \lambda^{2}, 0, z\right), \\
& y_{l}^{(2)}(z)=c N\left(4 l+2,2 \lambda, \lambda^{2}, 0, z\right) \ln z+\sum_{n \geq 0} d_{n} z^{n},
\end{aligned}
$$

where $N(\alpha, \beta, \gamma, \delta, z)$ is the Heun biconfluent function [3, 6], the constant

$$
c=\frac{1}{4 l+2}\left[d_{4 l+1} \lambda(4 l+1)-d_{4 l}\left(\lambda^{2}-4 l\right)\right],
$$

and the coefficient $d_{\nu}$ is given by the recurrence relation

$$
\begin{aligned}
& d_{-1}=0, \quad d_{0}=1 \\
& (\nu+2)(\nu-4 l) d_{\nu+2}-\lambda(2 \nu+1-4 l) d_{\nu+1}+\left[\lambda^{2}-2(\nu+1)+4 l+2\right] d_{\nu}=0 .
\end{aligned}
$$

The solution can be determined by the boundary condition (3.1).

The Heun biconfluent function has the expansion [3]

$$
N\left(4 l+2,2 \lambda, \lambda^{2}, 0, z\right)=\sum_{n \geq 0} \frac{A_{n}}{(4 l+3)_{n}} \frac{z^{n}}{n !},
$$

where the coefficient $A_{n}$ is given by

$$
\begin{aligned}
A_{0} & =1, \quad A_{1}=(4 l+3) \lambda, \\
A_{n+2} & =\lambda(4 l+2 n+5) A_{n+1}-(n+1)(4 l+n+3)\left[\lambda^{2}-(4 l+2 n+4)\right] A_{n},
\end{aligned}
$$

and $(a)_{n}=\Gamma(a+n) / \Gamma(a)$ is Pochhammer's symbol.

Obviously, only $f_{l}(z)=y_{l}^{(1)}(z)$ satisfies the boundary condition of the regular solution, eq. (3.1). By eq. (2.3) and $z=\sqrt{-2 i k r}$, we arrive at

$$
u_{l}(r)=A_{l}(-2 i k r)^{l+1} \exp \left(i\left(k r+\frac{\alpha}{k} \sqrt{r}\right)\right) N\left(4 l+2,2 \lambda, \lambda^{2}, 0, \sqrt{-2 i k r}\right) .
$$

This is the regular solution.

\section{Irregular solution}

The irregular solution is a solution satisfying the boundary condition at $r \rightarrow \infty$. At $r \rightarrow \infty$, the bound state and the scattering state require different boundary conditions.

\subsection{Scattering boundary condition}

The scattering boundary condition is determined by the asymptotic solution of the radial equation (2.2) at $r \rightarrow \infty$. For long-range potentials, the asymptotic behavior is determined by the potential, so different potentials give different scattering boundary conditions. As a comparison, for short-range potentials, the large-distance asymptotic behavior is determined by the centrifugal potential $l(l+1) / r^{2}$ which indeed comes from the kinetic energy rather 
than the external potential, so the asymptotic solution and, then, the scattering boundary condition, are the same for all short-range potentials.

To determine the scattering boundary condition, we first solve the asymptotic solution of the radial equation (2.2).

Writing the radial wave function $u_{l}(r)$ as

$$
u_{l}(r)=e^{h(r)} \exp ( \pm i k r)
$$

and substituting into the radial equation (2.2) give the equation of $h(r)$ :

$$
h^{\prime \prime}(r)+\left[h^{\prime}(r)\right]^{2} \pm 2 i k h^{\prime}(r)=\frac{l(l+1)}{r^{2}}-\frac{\alpha}{\sqrt{r}} .
$$

The leading-order contributions on the right-hand side and the left-hand side of eq. (4.2) must be the same order of magnitude, so we have

$$
\pm 2 i k h^{\prime}(r) \stackrel{r \rightarrow \infty}{\sim}-\frac{\alpha}{\sqrt{r}}
$$

This gives

$$
h(r) \sim \pm i \frac{\alpha}{k} \sqrt{r}
$$

Repeating the procedure in eq. (4.1) and taking the result given by eq. (4.4) into consideration, we write the radial wave function $u_{l}(r)$ as

$$
u_{l}(r)=e^{g(r)} \exp \left( \pm i\left(k r+\frac{\alpha}{k} \sqrt{r}\right)\right)
$$

and substitute into the radial equation (2.2). We then have the equation of $g(r)$ :

$$
g^{\prime \prime}(r)+\left[g^{\prime}(r)\right]^{2} \pm 2 i k g^{\prime}(r) \pm \frac{i \alpha}{k \sqrt{r}} g^{\prime}(r)=\frac{l(l+1)}{r^{2}}+\frac{\alpha^{2}}{4 k^{2} r} \pm \frac{i \alpha}{4 k r^{3 / 2}} .
$$

By the same reason, we have

$$
\pm 2 i k g^{\prime}(r) \stackrel{r \rightarrow \infty}{\sim} \frac{\alpha^{2}}{4 k^{2} r}
$$

This gives

$$
g(r) \sim \mp i \frac{\alpha^{2}}{8 k^{3}} \ln r .
$$

Again, repeating the above procedure, we write the radial wave function $u_{l}(r)$ as

$$
u_{l}(r)=e^{v(r)} \exp \left( \pm i\left(k r+\frac{\alpha}{k} \sqrt{r}-\frac{\alpha^{2}}{8 k^{3}} \ln r\right)\right)
$$

and substitute into the radial equation (2.2). We then have the equation of $v(r)$ :

$v^{\prime \prime}(r)+\left[v^{\prime}(r)\right]^{2} \pm 2 i k v^{\prime}(r) \pm \frac{i \alpha}{k \sqrt{r}} v^{\prime}(r) \mp \frac{i \alpha^{2}}{4 k^{3} r} v^{\prime}(r)=\frac{l(l+1)}{r^{2}}+\frac{\alpha^{4}}{64 k^{6} r^{2}} \mp \frac{i \alpha^{2}}{8 k^{3} r^{2}}-\frac{\alpha^{3}}{8 k^{4} r^{3 / 2}} \pm \frac{i \alpha}{4 k r^{3 / 2}}$. 
By the same reason, we have

$$
\pm 2 i k v^{\prime}(r)=-\frac{\alpha^{3}}{8 k^{4} r^{3 / 2}} \pm \frac{i \alpha}{4 k r^{3 / 2}}
$$

It can be directly seen that

$$
v(r) \sim \frac{1}{\sqrt{r}}
$$

This means that when $r \rightarrow \infty$, the contribution of $v(r)$ vanishes and does not need to be taken into account.

We can now write down the asymptotic radial function:

$$
u_{l}^{\infty}(r)=\exp \left( \pm i\left(k r+\frac{\alpha}{k} \sqrt{r}-\frac{\alpha^{2}}{8 k^{3}} \ln r\right)\right)
$$

The scattering boundary condition, then, can be written as

$$
\lim _{r \rightarrow \infty} \exp \left( \pm i\left(k r+\frac{\alpha}{k} \sqrt{r}-\frac{\alpha^{2}}{8 k^{3}} \ln r\right)\right) u_{l}(r)=1 .
$$

It is worth to compare the scattering boundary condition (4.14) with the scattering boundary condition of the Coulomb potential, $\lim _{r \rightarrow \infty} \exp \left( \pm i\left(k r-\frac{\alpha}{2 k} \ln r\right)\right) u_{l}(k r)=1$ [4].

\subsection{Irregular solution}

With the scattering boundary condition at $r \rightarrow \infty$, eq. (4.14), we can now determine the irregular solution which is the solution satisfying the boundary condition at $r \rightarrow \infty$.

The biconfluent Heun equation (2.4), which relates the radial equation by the substitution (2.3), has two linearly independent solutions satisfying the scattering boundary condition (4.14) [3]

$$
\begin{aligned}
& B_{l}^{+}\left(4 l+2,2 \lambda, \lambda^{2}, 0, z\right)=z^{\left(\lambda^{2}-4 l-4\right) / 2} \sum_{n \geq 0} \frac{a_{n}}{z^{n}} \\
& H_{l}^{+}\left(4 l+2,2 \lambda, \lambda^{2}, 0, z\right)=z^{-\left(\lambda^{2}+4 l+4\right) / 2} e^{2 \lambda z+z^{2}} \sum_{n \geq 0} \frac{e_{n}}{z^{n}}
\end{aligned}
$$

where $B_{l}^{+}(\alpha, \beta, \gamma, \delta, z)$ and $H_{l}^{+}(\alpha, \beta, \gamma, \delta, z)$ are another two kinds of the biconfluent Heun functions, which is different from the biconfluent Heun functions mentioned above, and the coefficients $a_{n}$ and $e_{n}$ are given by

$$
\begin{aligned}
& a_{0}=1, a_{1}=-\frac{1}{2} \lambda\left(\lambda^{2}+1\right) \\
& 2(n+2) a_{n+2}+\left[\lambda^{3}+(2 n+3) \lambda\right] a_{n+1}+\left[\frac{1}{4} \lambda^{4}-(n+1) \lambda^{2}+n(n+2)-4 l(l+1)\right] a_{n}=0
\end{aligned}
$$


and

$e_{0}=1, e_{1}=\frac{1}{2} \lambda\left(\lambda^{2}-1\right)$

$2(n+2) e_{n+2}-\left[\lambda^{3}-(2 n+3) \lambda\right] e_{n+1}-\left[\frac{1}{4} \lambda^{4}+(n+1) \lambda^{2}+n(n+2)-4 l(l+1)\right] e_{n}=0$.

These two solutions both are irregular solutions.

\section{Bound state and scattering state}

In order to achieve the bound-state and scattering-state solutions, we first express the regular solution (3.10) as a linear combination of the two irregular solutions [3]:

$$
\begin{aligned}
N\left(4 l+2,2 \lambda, \lambda^{2}, 0, \sqrt{-2 i k r}\right) & =K_{1}\left(4 l+2,2 \lambda, \lambda^{2}, 0\right) B_{l}^{+}\left(4 l+2,2 \lambda, \lambda^{2}, 0, \sqrt{-2 i k r}\right) \\
& +K_{2}\left(4 l+2,2 \lambda, \lambda^{2}, 0\right) H_{l}^{+}\left(4 l+2,2 \lambda, \lambda^{2}, 0, \sqrt{-2 i k r}\right),
\end{aligned}
$$

where $K_{1}\left(4 l+2,2 \lambda, \lambda^{2}, 0\right)$ and $K_{2}\left(4 l+2,2 \lambda, \lambda^{2}, 0\right)$ are the coefficients of combination. Then by eq. (3.10) and eqs. (4.15) and (4.16), we have

$$
\begin{aligned}
u_{l}(r) & =A_{l} K_{1}\left(4 l+2,2 \lambda, \lambda^{2}, 0\right)(-2 i k)^{-i \alpha^{2} /\left(8 k^{3}\right)} \exp \left(i\left(k r+\frac{\alpha}{k} \sqrt{r}-\frac{\alpha^{2}}{8 k^{3}} \ln r\right)\right) \sum_{n \geq 0} \frac{a_{n}}{(-2 i k r)^{n / 2}} \\
& +A_{l} K_{2}\left(4 l+2,2 \lambda, \lambda^{2}, 0\right)(-2 i k)^{i \alpha^{2} /\left(8 k^{3}\right)} \exp \left(-i\left(k r+\frac{\alpha}{k} \sqrt{r}-\frac{\alpha^{2}}{8 k^{3}} \ln r\right)\right) \sum_{n \geq 0} \frac{e_{n}}{(-2 i k r)^{n / 2}} .
\end{aligned}
$$

Here, we use the expansions (4.15) and (4.16) for convenience in analyzing the asymptotic behavior of the solution.

\subsection{Bound state}

By analytical continuation $k$ to the complex plane, we can consider $k$ along the positive imaginary axis. On the positive imaginary axis, we define

$$
k=i \kappa, \quad \kappa>0 .
$$

Then eq. (5.2), with $\lambda=\alpha / \sqrt{2 \kappa^{3}}$, becomes

$$
\begin{aligned}
u_{l}(r) & =A_{l} K_{1}\left(4 l+2,2 \lambda, \lambda^{2}, 0\right) \exp \left(-\kappa r+\frac{\alpha}{\kappa} \sqrt{r}\right)(2 \kappa r)^{\alpha^{2} /\left(8 \kappa^{3}\right)} \sum_{n \geq 0} \frac{a_{n}}{(2 \kappa r)^{n / 2}} \\
& +A_{l} K_{2}\left(4 l+2,2 \lambda, \lambda^{2}, 0\right) \exp \left(\kappa r-\frac{\alpha}{\kappa} \sqrt{r}\right)(2 \kappa r)^{-\alpha^{2} /\left(8 \kappa^{3}\right)} \sum_{n \geq 0} \frac{e_{n}}{(2 \kappa r)^{n / 2}} .
\end{aligned}
$$


It can be directly seen that the first term vanishes when $r \rightarrow \infty$ due to the factor $\exp (-\kappa r+\alpha \sqrt{r} / \kappa)$ and, in contrast, the second term diverges when $r \rightarrow \infty$ due to the factor $\exp (\kappa r-\alpha \sqrt{r} / \kappa)$. Clearly, when $K_{2}\left(4 l+2,2 \lambda, \lambda^{2}, 0\right)$ equals zero, the solution $u_{l}(r)$ will satisfy the bound-state boundary condition. That is to say, the zeroes of the coefficient $K_{2}\left(4 l+2,2 \lambda, \lambda^{2}, 0\right)$ corresponds to the bound state. This implies that the zeroes of $K_{2}\left(4 l+2,2 \lambda, \lambda^{2}, 0\right)$ on the imaginary axis determines the spectrum of the bound state. Consequently, the spectrum of the bound-state eigenvalue is determined by

$$
K_{2}\left(4 l+2, \frac{2 \alpha}{\sqrt{2 \kappa^{3}}}, \frac{\alpha^{2}}{2 \kappa^{3}}, 0\right)=0
$$

where [3]

$$
K_{2}(\alpha, \beta, \gamma, \delta)=\frac{\Gamma(1+\alpha)}{\Gamma((\alpha-\gamma) / 2) \Gamma(1+(\alpha+\gamma) / 2)} J_{1+(\alpha+\gamma) / 2}\left(\frac{1}{2}(\alpha+\gamma), \beta, \frac{1}{2}(3 \alpha-\gamma), \delta+\frac{1}{2} \beta(\gamma-\alpha)\right)
$$

with

$$
J_{\lambda}(\alpha, \beta, \gamma, \delta)=\int_{0}^{\infty} x^{\lambda-1} e^{-x^{2}-\beta x} N(\alpha, \beta, \gamma, \delta, x) d x
$$

Eq. (5.5) is an implicit expression of the bound-state spectrum.

The bound-state eigenfunction is then

$$
u_{l}(r)=C \exp \left(-\kappa r+\frac{\alpha}{\kappa} \sqrt{r}\right)(2 \kappa r)^{\alpha^{2} /\left(8 \kappa^{3}\right)} \sum_{n \geq 0} \frac{a_{n}}{(2 \kappa r)^{n / 2}}
$$

where the eigenvalue $\kappa$ is given by eq. (5.5) and $C$ is a normalization constant.

\section{$5.2 \quad$ Scattering state}

It is known that the singularity on the positive imaginary axis of the $S$-matrix corresponds to the spectrum of bound states [2]. While, as pointed above, the zeroes on the positive imaginary of the coefficient $K_{2}\left(4 l+2,2 \lambda, \lambda^{2}, 0\right)$ correspond to the spectrum of bound states. Therefore, the zeroes on the positive imaginary of $K_{2}\left(4 l+2,2 \lambda, \lambda^{2}, 0\right)$ are just the singularities of the $S$-matrix. Considering that the $S$-matrix

$$
S_{l}=e^{2 i \delta_{l}}
$$

is unitary, we then have

$$
S_{l}=\frac{K_{2}^{*}\left(4 l+2,2 \lambda, \lambda^{2}, 0\right)}{K_{2}\left(4 l+2,2 \lambda, \lambda^{2}, 0\right)}=\frac{K_{2}\left(4 l+2,-2 i \lambda,-\lambda^{2}, 0\right)}{K_{2}\left(4 l+2,2 \lambda, \lambda^{2}, 0\right)},
$$

where $K_{2}^{*}\left(4 l+2, \frac{2 \alpha}{\sqrt{2 i k^{3}}}, \frac{\alpha^{2}}{2 i k^{3}}, 0\right)=K_{2}\left(4 l+2, \frac{2 \alpha}{\sqrt{-2 i k^{3}}},-\frac{\alpha^{2}}{2 i k^{3}}, 0\right)$ is used.

By the $S$-matrix (5.10), we can construct the scattering wave function. The scattering wave function can be generally written as $u_{l}(r)=A_{l}\left[(-1)^{l+1} u_{\text {in }}+S_{l} u_{\text {out }}\right]$ with $u_{\text {in }}=u_{\text {out }}^{*}$, where $u_{i n}$ and $u_{\text {out }}$ are the radially ingoing and outgoing waves, respectively [2]. 
Now we can write down the scattering wave function

$$
\begin{aligned}
u_{l}(r) & =A_{l}\left[(-1)^{l+1} \exp \left(-i\left(k r+\frac{\alpha}{k} \sqrt{r}-\frac{\alpha^{2}}{8 k^{3}} \ln r\right)\right) \sum_{n \geq 0} \frac{e_{n}}{(-2 i k r)^{n / 2}}\right. \\
& \left.+\frac{K_{2}\left(4 l+2,2 i \lambda,-\lambda^{2}, 0\right)}{K_{2}\left(4 l+2,2 \lambda, \lambda^{2}, 0\right)} \exp \left(i\left(k r+\frac{\alpha}{k} \sqrt{r}-\frac{\alpha^{2}}{8 k^{3}} \ln r\right)\right) \sum_{n \geq 0} \frac{e_{n}^{*}}{(2 i k r)^{n / 2}}\right] .
\end{aligned}
$$

At $r \rightarrow \infty$, we have the large-distance asymptotics

$$
\begin{aligned}
& u_{l}(r) \stackrel{r \rightarrow \infty}{\sim} A_{l} i^{l}\left[-i^{l} \exp \left(-i\left(k r+\frac{\alpha}{k} \sqrt{r}-\frac{\alpha^{2}}{8 k^{3}} \ln r\right)\right)\right. \\
& \left.+i^{-l} \frac{K_{2}\left(4 l+2,-2 i \lambda,-\lambda^{2}, 0\right)}{K_{2}\left(4 l+2,2 \lambda, \lambda^{2}, 0\right)} \exp \left(i\left(k r+\frac{\alpha}{k} \sqrt{r}-\frac{\alpha^{2}}{8 k^{3}} \ln r\right)\right)\right], \\
& =A_{l} i^{l} e^{i \delta_{l}} \sin \left(k r+\frac{\alpha}{k} \sqrt{r}-\frac{\alpha^{2}}{8 k^{3}} \ln r+\delta_{l}-\frac{l \pi}{2}\right) .
\end{aligned}
$$

The scattering phase shift here, by eqs. (5.9) and (5.10), is

$$
\delta_{l}=-\arg K_{2}\left(4 l+2,2 \lambda, \lambda^{2}, 0\right) .
$$

\section{Conclusion}

In this paper, we present an exact solution of the spherical symmetry inverse-power potential $-\alpha / \sqrt{r}$.

It is worthy to emphasized here that we can put a stronger definition for long-range potentials: if the asymptotic behavior of a potential is the same as that of short-range potentials, i.e., large-distance asymptotics is $e^{ \pm i k r}$, the potential is a short-range potential. Under this definition, the inverse-power potential $V(r) \sim 1 / r^{s}$ with $0<s<1$ is a longrange potential, but $V(r) \sim 1 / r^{s}$ with $1<s<2$ is a short-range potential. Even under such a stronger definition, the inverse-power potential $\alpha / \sqrt{r}$ is still a long-range potential.

The three-dimensional inverse square root potential is a long-range potential. The difficulty of the study of long-range-potential scattering is that the scattering boundary condition is determined by the external potential rather than the centrifugal potential as that in short-range-potential scattering. Scattering boundary conditions for different largedistance potentials are different. To seek the scattering boundary condition for long-range potentials needs to first determine the large-distance asymptotic solution. There are some discussions on the long-range-potential scattering [7-9]. Scattering by combination of known long-range and unknown short-range potentials is studied by the renormalization-group method [10]. The late-time dynamics of the wave equation with a long-range potential is discussed in ref. [11]. The spectral property of scattering matrix of the Schrödinger operator with a long-range potential is considered in ref. [12]. Scattering on black holes is essentially a kind of long-range scattering. [13, 14]. The partial derivative of scattering phase shifts with respect to wave number $k$ for long-range potentials is given in ref. [4]. There are many 
efforts on seeking the scattering phase shift $[15,16]$. There are also studies on the orbit in power-law potentials [17], the screened Coulomb potential, and the isotropic harmonic oscillator [18]. The number of bound states [19, 20], the number of eigenstates [21, 22], the conditions for the existence of bound states $[23,24]$ are also important problems.

\section{Acknowledgments}

We are very indebted to Dr G. Zeitrauman for his encouragement. This work is supported in part by NSF of China under Grant No. 11575125 and No. 11375128.

\section{References}

[1] T. Liu, W.-D. Li, and W.-S. Dai, Scattering theory without large-distance asymptotics, Journal of High Energy Physics 2014 (2014), no. 6 1-12.

[2] C. J. Joachain, Quantum collision theory, .

[3] A. Ronveaux and F. M. Arscott, Heun's differential equations. Oxford University Press, 1995.

[4] W. Romo and S. Valluri, A study of the momentum dependence of the phase shift for finite range and coulomb potentials and its possible applications, Nuclear Physics A 636 (1998), no. 4 467-484.

[5] L. E. Ballentine, Quantum mechanics: a modern development. World scientific, 1998.

[6] S. Slavyanov and W. Lay, Special functions: a unified theory based on singularities. Oxford University Press, 2000.

[7] V. Enss, Asymptotic completeness for quantum-mechanical potential scattering: Ii. singular and long-range potentials, Annals of Physics 119 (1979), no. 1 117-132.

[8] B. R. Levy and J. B. Keller, Low-energy expansion of scattering phase shifts for long-range potentials, Journal of Mathematical Physics 4 (1963), no. 1 54-64.

[9] O. Hinckelmann and L. Spruch, Low-energy scattering by long-range potentials, Physical Review A 3 (1971), no. 2642.

[10] T. Barford and M. C. Birse, Renormalization group approach to two-body scattering in the presence of long-range forces, Physical Review C 67 (2003), no. 6064006.

[11] S. Hod, Scattering by a long-range potential, Journal of High Energy Physics 2013 (2013), no. 9 1-11.

[12] D. Yafaev, The scattering amplitude for the schrödinger equation with a long-range potential, Communications in mathematical physics 191 (1998), no. 1 183-218.

[13] Y. Stadnik, G. Gossel, V. Flambaum, and J. Berengut, Resonant scattering of light in a near-black-hole metric, The European Physical Journal C 73 (2013), no. 11 1-7.

[14] V. Flambaum, G. Gossel, and G. Gribakin, Dense spectrum of resonances and particle capture in a near-black-hole metric, Physical Review D 85 (2012), no. 8084027.

[15] H. Pang, W.-S. Dai, and M. Xie, Relation between heat kernel method and scattering spectral method, The European Physical Journal C 72 (2012), no. 5 1-13.

[16] W.-D. Li and W.-S. Dai, Heat-kernel approach for scattering, The European Physical Journal $C 75$ (2015), no. 6. 
[17] A. K. Grant and J. L. Rosner, Classical orbits in power-law potentials, American Journal of Physics 62 (1994), no. 4 310-314.

[18] Z.-B. Wu and J.-Y. Zeng, Dynamical symmetry of screened coulomb potential and isotropic harmonic oscillator, Physical Review A 62 (2000), no. 3032509.

[19] V. Bargmann, On the number of bound states in a central field of force, Proceedings of the National Academy of Sciences 38 (1952), no. 11 961-966.

[20] F. Calogero, Upper and lower limits for the number of bound states in a given central potential, Communications in Mathematical Physics 1 (1965), no. 1 80-88.

[21] W.-S. Dai and M. Xie, The number of eigenstates: counting function and heat kernel, Journal of High Energy Physics 2009 (2009), no. 02033.

[22] W.-S. Dai and M. Xie, An approach for the calculation of one-loop effective actions, vacuum energies, and spectral counting functions, Journal of High Energy Physics 2010 (2010), no. 6 $1-29$.

[23] F. Brau, Necessary and sufficient conditions for existence of bound states in a central potential, Journal of Physics A: Mathematical and General 36 (2003), no. 389907.

[24] F. Brau, Sufficient conditions for the existence of bound states in a central potential, Journal of Physics A: Mathematical and General 37 (2004), no. 266687. 